\title{
Implantação de lente intraocular com uma alça amputada: proposta para o tratamento cirúrgico da subluxação do cristalino
}

\author{
Intraocular lens implantation with one loop haptic amputed: a new propose to \\ the subluxation lens surgical treatment
}

\author{
Marcelo Ventura ${ }^{1}$ \\ Daniela Endriss ${ }^{2}$
}

\section{RESUMO}

Objetivo: Avaliar os resultados pós-operatórios da subluxação congênita do cristalino, corrigida por uma nova abordagem cirúrgica. Métodos: Foram estudados 21 olhos de 13 pacientes, portadores de subluxação não traumática do cristalino submetidos à cirurgia na Fundação Altino Ventura, no período de abril de 1999 a abril de 2004. A idade média foi de 8,7 $\pm 5,4$ anos, e o tempo médio de seguimento foi 21,5 $\pm 19,3$ meses. Os pacientes foram submetidos à facoaspiração, implante do anel endocapsular e lente intraocular (LIO). Uma das alças da LIO foi amputada e apoiada sobre o anel, no interior do saco capsular, centralizando a LIO. Resultados: Houve melhora da acuidade visual (AV) em todos os casos, e redução significante do equivalente esférico e componente esférico comparando-se a refração pré e pós-operatória $(\mathrm{p}<0,01)$. Não houve diferença entre o componente cilíndrico pré e pós-operatório $(\mathrm{p}=0,71)$. Opacificação da cápsula posterior do cristalino foi a complicação encontrada em $71,4 \%$ dos casos. Foi realizada capsulotomia posterior precoce nestes pacientes sem intercorrências. Conclusão: Implante de LIO com uma alça amputada e apoiada sobre o anel endocapsular é uma opção no tratamento cirúrgico da subluxação congênita do cristalino, podendo-se observar centralização da LIO e melhora da AV no pós-operatório.

Descritores: Subluxação do cristalino; Implante de prótese; Síndrome de Marfan; Homocistinúria; Lentes intraoculares; Ectopia do cristalino/cirurgia; Procedimentos cirúrgicos oftalmológicos; Acuidade visual

\section{INTRODUÇÃO}

A subluxação do cristalino pode ocorrer como manifestação de doença sistêmica como na síndrome de Marfan, homocistinúria, ou na síndrome de Weil-Marchesani, ou em associação com outras doenças oculares, entre elas a megalocórnea ${ }^{(1-9)}$. A subluxação do cristalino idiopática e a ectopia familiar simples ocorre nos casos em que nenhuma outra anormalidade ocular ou sistêmica é observada ${ }^{(1,7)}$. Pacientes com subluxação congênita, classicamente por síndrome de Marfan, apresentam uma gama de achados oculares associados, que vão de alterações estruturais de ângulo à maior incidência de degenerações retinianas periféricas, com risco de ruptura e descolamento de retina regmatogênico ${ }^{(1-3)}$.

O cristalino ectópico pode causar alterações no estado refrativo do olho e redução da acuidade visual ${ }^{(3)}$. A miopia lenticular resulta do aumento da curvatura do cristalino e se deve basicamente ao afrouxamento da zônula. Além 
da miopia, o astigmatismo gerado pela periferia do cristalino contribui para as distorções da imagem. Os erros de refração não corrigidos na infância podem provocar acentuada ambliopia, principalmente quando a subluxação do cristalino ocorre numa idade precoce ${ }^{(1-4,7,9)}$.

A ectopia do cristalino vem sendo tratada com cirurgia há longo tempo e discute-se o momento ideal da indicação e da técnica a ser utilizada ${ }^{(3,5)}$. A perda significante de suporte zonular dificulta o procedimento cirúrgico e a implantação da LIO. Em 1993, alguns autores propuseram o implante de um anel endocapsular com estabilização intra e pós-operatória do saco capsular e da $\mathrm{LIO}^{(2,9-12)}$. O anel endocapsular ajuda a manter o contorno circular do saco capsular, evitando a descentração da LIO, exceto em alguns casos com extensa fragilidade zonular, em que muitas vezes não é possível adequada centralização da cápsula e da $\mathrm{LIO}^{(2,9-13)}$. Outros autores em 1998 acrescentaram ao anel endocapsular uma alça para fixação do saco capsular com manutenção de sua integridade, tornando ainda mais fisiológica uma cirurgia que evoluiu muito nos últimos cinco anos ${ }^{(6,10)}$.

Na Fundação Altino Ventura e Hospital de Olhos de Pernambuco vêm sendo desenvolvida, desde 1999, uma nova proposta cirúrgica para os pacientes com subluxação do cristalino, possibilitando o implante e centralização da LIO no saco capsular. A técnica de implante da LIO com uma alça amputada, apoiada no anel endocapsular, foi desenvolvida por um dos autores (MV), e tem possibilitado a centralização pósoperatória da LIO em casos de subluxação do cristalino, em pacientes jovens. O objetivo deste estudo é descrever a técnica cirúrgica proposta e os resultados cirúrgicos em 13 pacientes portadores de subluxação não traumática do cristalino.

\section{MÉTODOS}

Foi realizado um estudo retrospectivo, de uma série de casos de pacientes portadores de subluxação não traumática do cristalino, submetidos a tratamento cirúrgico no período de abril de 1999 a abril de 2004. Foram incluídos no estudo 13 pacientes ( 21 olhos), com idade variando de 3 a 18 anos, média de 8,7 anos e desvio padrão (dp) $\pm 5,4$ anos, operados na Fundação Altino Ventura, pelo mesmo cirurgião (MV), e mesma técnica cirúrgica.

Os pacientes foram submetidos a avaliação oftalmológica pré e pós-operatória, que incluiu: acuidade visual com melhor correção (tabela de Snellen ou de figuras), biomicroscopia, refração estática e dinâmica, mapeamento de retina, tonometria e biometria.

\section{Técnica cirúrgica}

Todos os pacientes foram submetidos à anestesia geral, com exceção de um paciente adulto (18 anos), que foi realizada anestesia local peri-bulbar. Após dilatação da pupila com colírio de tropicamida $1 \%$ e ciclopentolato $1 \%$, realizou-se abertura superior da conjuntiva, seguindo-se de incisão escleral tunelizada a aproximadamente $1,5 \mathrm{~mm}$ do limbo e preenchimento da câmara anterior com substância viscoelástica (metilcelulose 2\%). A capsulorrexe foi de aproximadamente $3 \mathrm{~mm}$, descentrada em direção à subluxação ou às 12 horas, confeccionada com pinça Utrata (Figura 1). Implantou-se o anel endocapsular no saco capsular (Mediphacos 12-10) com injetor ou manualmente, de forma que as extremidades deste permaneçam próximas das 12 horas, fora da área de maior fragilidade zonular. O cortex e núcleo cristaliniano foram aspirados, utilizando-se ponteira de irrigação/aspiração (Figuras 2 e 3). Em crianças utilizamos LIO rígida de polimetilmetacrilato (PMMA), com 6,5 $\mathrm{mm}$ na parte óptica e 13,5 $\mathrm{mm}$ de alça a alça (Mediphacos). Amputou-se uma das alças da LIO com tesoura, deixando um pequeno coto de aproximadamente $1 \mathrm{~mm}$ para apoiar a LIO no anel endocapsular, na posição desejada (Figura 4). Implantou-se no saco capsular, inicialmente a alça amputada e posteriormente o lado da alça íntegra, deslocando a LIO dentro do saco. A LIO foi posicionada para que o coto da alça

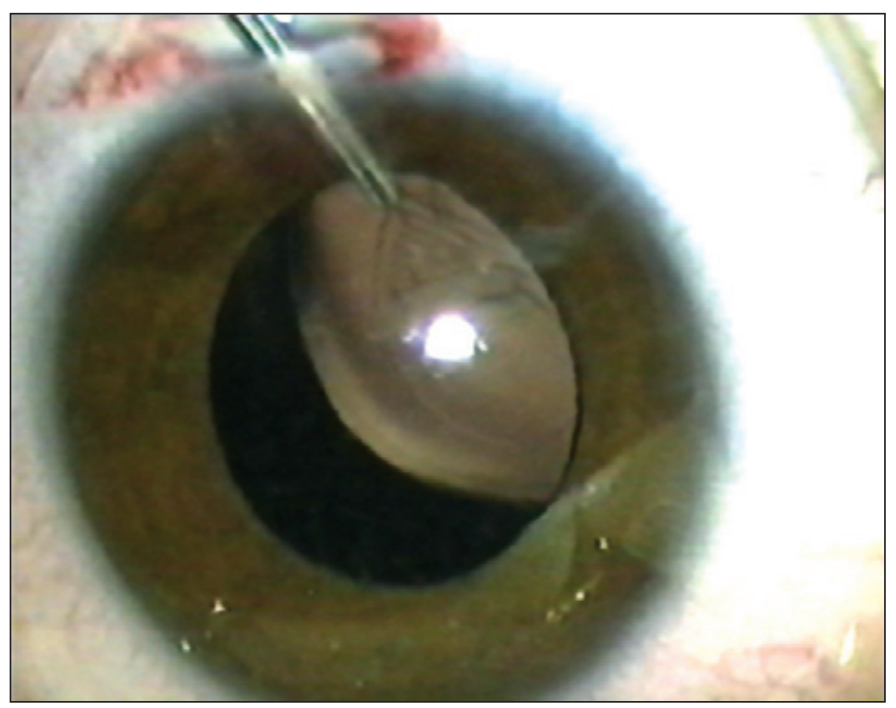

Figura 1 - Capsulorrexe descentrada

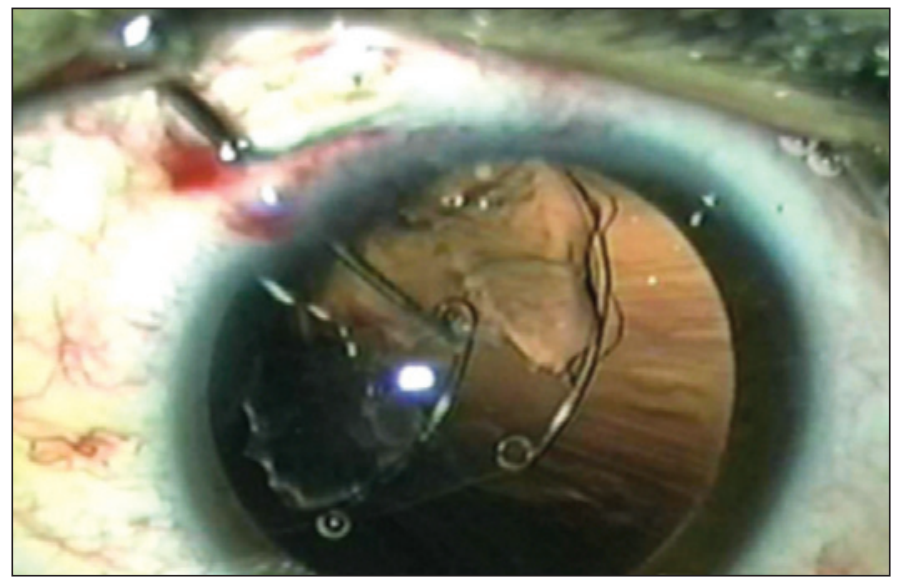

Figura 2 - Implante do anel endocapsular 
amputada apoiasse no anel endocapsular na área de maior subluxação, próximo às 8 horas, permitindo a descentralização da parte óptica da LIO para o eixo visual (Figuras 5 e 6). Não foram observadas complicações durante o implante da LIO, como ruptura do saco capsular pelo coto da alça, provavelmente devido à grande elasticidade destas cápsulas. Não utilizamos esta técnica em casos de subluxação do cristalino maior do que 240 graus, ou com presença de vítreo na câmara anterior pré ou per-operatoriamente. A incisão cirúrgica foi suturada com fio Nylon 10-0 e realizado injeção subconjuntival de dexametasona e gentamicina.

No pós-operatório foram prescritos colírios de prednisolona $1 \%$ de $3 / 3$ horas, com diminuição gradativa até 30 dias, antibiótico (ciprofloxacina 0,3\%) de $6 / 6$ horas por duas semanas e midriático (tropicamida 1\%) de 12/12 horas por 10 dias. Os pacientes foram avaliados no $1^{\circ}, 7^{\circ}, 15^{\circ}$ e $30^{\circ}$ dias de pósoperatório, e posteriormente de acordo com a evolução. Foram prescritos óculos multifocais entre sete e 15 dias após a cirurgia.

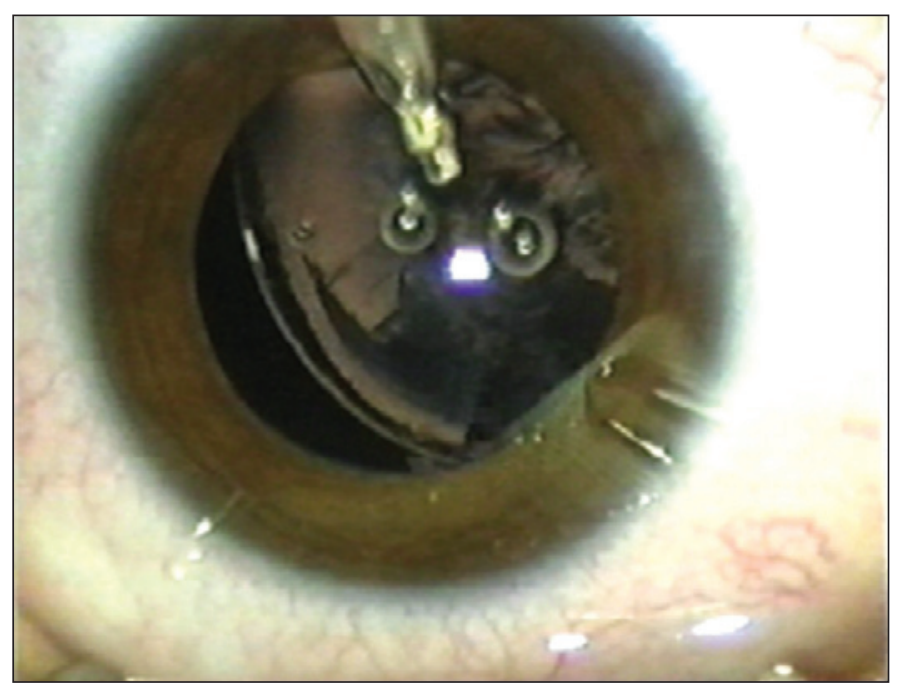

Figura 3 - Aspiração das massas corticais

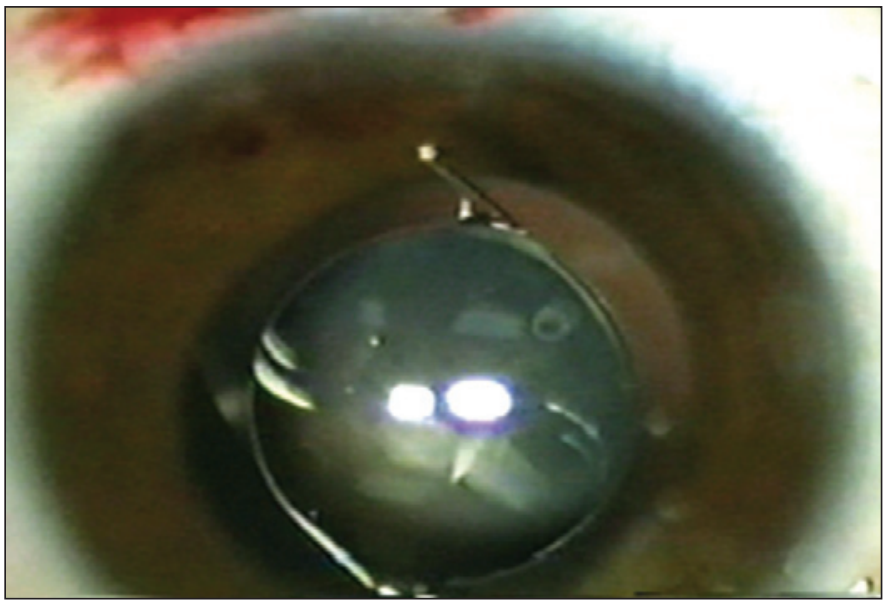

Figura 4 - Amputação da alça da lente intraocular
Os dados foram tabulados através de frequências absolutas e relativas, e apresentados em forma de tabelas e gráficos. Foi utilizado o teste t pareado, considerando-se como significante o valor de $\mathrm{p}<0,05$.

\section{RESULTADOS}

Do total de 13 pacientes operados, 10 (76,9\%) eram do sexo masculino. O tempo de seguimento pós-operatório variou de seis meses a 4,7 anos, média de 2,3 anos $\pm 1,5$ anos. $O$ comprimento axial dos olhos variou de 22,3 a $27,9 \mathrm{~mm}$, com média de $25,1 \pm 1,8 \mathrm{~mm}$. O poder da LIO implantada foi em média de 18,6 $\pm 5,9 \mathrm{D}$, variando de 7 a 27 dioptrias (D).

Em todos os pacientes houve melhora da acuidade visual corrigida no pós-operatório (Tabela 1), e observou-se melhora

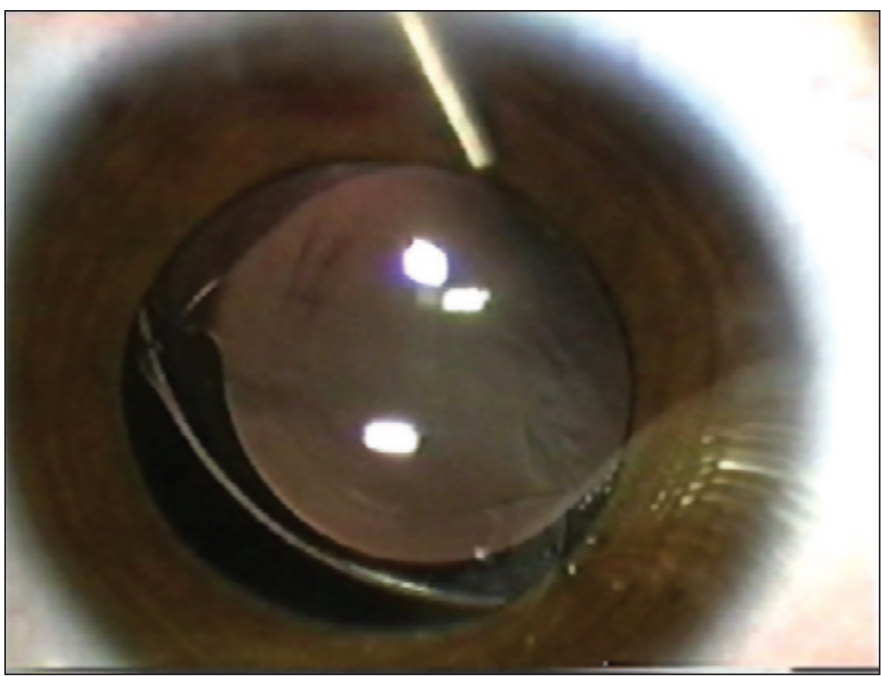

Figura 5 - Lente intraocular com a alça amputada apoiada sobre o anel endocapsular

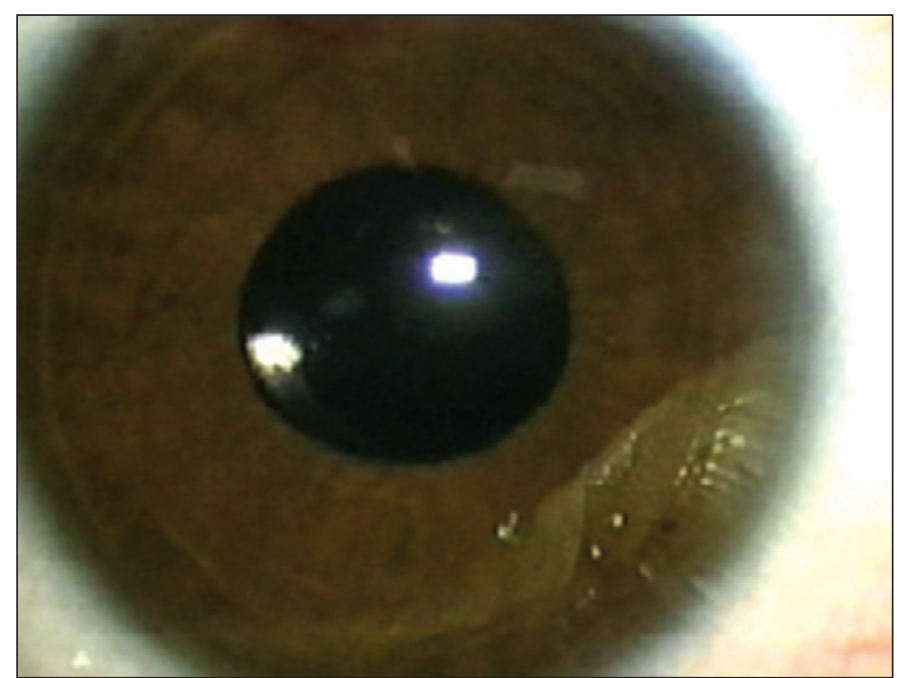

Figura 6 - Lente intraocular centralizada, aspecto final 
de cinco ou mais linhas na acuidade visual (Tabela de Snellen) em 9 olhos (42,9\%) (Gráfico 1).

Houve redução estatisticamente significante do equivalente esférico e do componente esférico comparando-se os valores pré e pós-operatórios ( $\mathrm{p}<0,01)$ (Tabelas 2 e 3 ). Não foi observada diferença significativa entre o componente cilíndrico pré e pós-operatório ( $\mathrm{p}=0,71)$ (Tabela 4$)$.

Não houve complicação intraoperatória. No pós-operatório, foi observada opacificação da cápsula posterior do cristalino (OCP) em 15 olhos (71,4\%), ocorrendo em média 1,2 \pm 1,0 anos após a cirurgia (variou de 3 meses a 3,4 anos). Dez olhos $(47,6 \%)$ foram submetidos à capsulotomia posterior com Nd:YAG laser, e 2 olhos $(9,5 \%)$ à capsulotomia posterior cirúrgica. Não houve complicações secundárias após a capsulotomia posterior.

\section{DISCUSS ÃO}

O tratamento da subluxação do cristalino, principalmente em crianças, é um desafio para a oftalmologia atual ${ }^{(2,6,9,13)}$. Mesmo com cuidadosa refração, nem sempre é possível obter visão satisfatória, com correção para fácico ou afácico, e a correção cirúrgica, muitas vezes, é a única alternativa viável para a melhora da acuidade visual e prevenção da ambliopia ${ }^{(3)}$. A técnica cirúrgica utilizada varia muito de caso para caso, na dependência de haver luxação, lesão zonular e hérnia de vítreo ${ }^{(5)}$.

Na última década, com o avanço da técnica de facectomia, da biocompatibilidade e desenho das lentes intraoculares e

Tabela 1. Acuidade visual com melhor correção pré e pós-operatória (Tabela de Snellen)

\begin{tabular}{|lcccc|} 
& \multicolumn{4}{c}{$\begin{array}{c}\text { Acuidade visual corrigida } \\
\text { Exame }\end{array}$} \\
\cline { 2 - 5 } & $\geq 20 / 40$ & $20 / 50-20 / 150$ & $\leq 20 / 200$ & Total \\
Pré-operatório & $0(0,0)$ & $14(66,7)$ & $7(33,3)$ & $21(100)$ \\
Pós-operatório & $12(57,1)$ & $8(38,1)$ & $1(4,8)$ & $21(100)$ \\
\hline
\end{tabular}

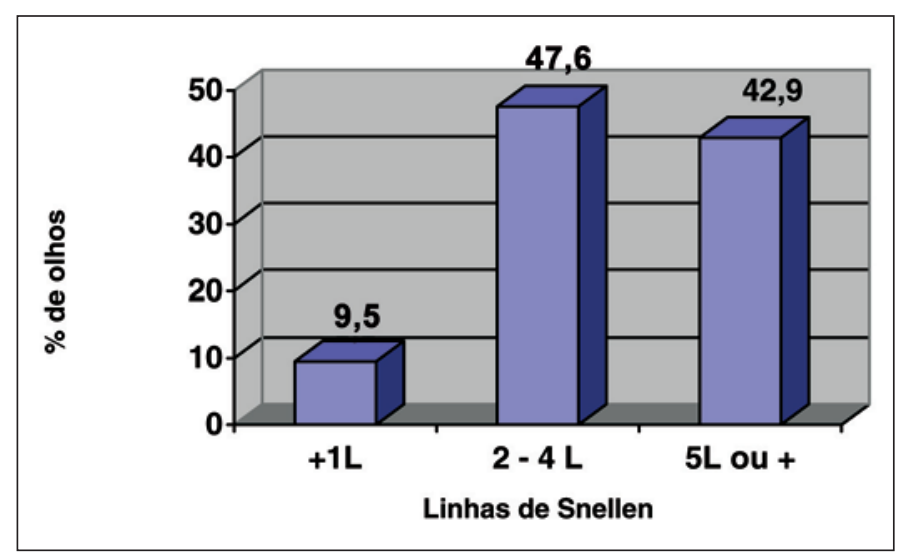

Gráfico 1 - Melhora da acuidade visual pós-operatória expressa em ganhos de linhas de visão (Tabela de Snellen) dos recursos disponíveis como adjuvantes da cirurgia, como o anel expansor endocapsular, a facectomia com implante primário de LIO em olhos com cristalino subluxado passou a ser uma opção viável(2). O implante de LIO em crianças elimina a anisometropia e aniseiconia que ocorrem particularmente nos casos unilaterais, evitando a ambliopia e estrabismo. Além disso, este procedimento evita o uso de lentes de contato que é bastante problemático em crianças, devido ao risco de infecção, à facilidade de perda e necessidade de grande colaboração dos pais ${ }^{(3,8)}$.

O implante de LIO em crianças com subluxação do cristalino apresenta um potencial de complicações pós-operatórias devido a características próprias do olho destes pacientes: proliferação epitelial secundária e contração da cápsula são mais pronunciadas, e o grau de diálise zonular, em pacientes com subluxação precoce, pode ser mais acentuado que em adultos $^{(3,14)}$. Os mecanismos responsáveis pela contração capsular e excessiva fibrose ainda não são bem conhecidos. A metaplasia das células epiteliais residuais e fibrose contribuem para a contração capsular, e uma resposta ainda mais intensa é encontrada nos casos de fragilidade do suporte zonular ${ }^{(15)}$. A combinação de severa proliferação epitelial e intensa fragilidade zonular pode levar à descentração da $\mathrm{LIO}^{(15)}$. No entanto, ao longo do seguimento destes pacientes, os autores observaram melhora da estabilização do conjunto formado pelo saco capsular, anel endocapsular e LIO, possivelmente devido à fibrose pós-operatória.

\begin{tabular}{|c|c|c|c|}
\hline \multirow[t]{2}{*}{ Exame } & \multicolumn{3}{|c|}{ Equivalente esférico (D) } \\
\hline & Média $\pm \mathrm{dp}$ & Mínimo & Máximo \\
\hline Pré-operatório & $-0,4 \pm 11,0$ & $-12,7$ & $+13,5$ \\
\hline Pós-operatório & $-0,6 \pm 1,5$ & $-3,6$ & $+2,2$ \\
\hline \multicolumn{4}{|c|}{$d p=$ desvio padrão; $p<0,01$} \\
\hline
\end{tabular}

\begin{tabular}{|c|c|c|c|}
\hline \multirow[t]{2}{*}{ Exame } & \multicolumn{3}{|c|}{ Componente esférico (D) } \\
\hline & Média $\pm \mathrm{dp}$ & Mínimo & Máximo \\
\hline Pré-operatório & $1,7 \pm 10,7$ & $-12,7$ & $+14,0$ \\
\hline Pós-operatório & $0,3 \pm 1,4$ & $-2,7$ & $+3,5$ \\
\hline \multicolumn{4}{|c|}{$d p=$ desvio padrão; $p<0,01$} \\
\hline
\end{tabular}

\begin{tabular}{|c|c|c|c|}
\hline \multirow[t]{2}{*}{ Exame } & \multicolumn{3}{|c|}{ Astigmatismo refratométrico (D) } \\
\hline & Média $\pm \mathrm{dp}$ & Mínimo & Máximo \\
\hline Pré-operatório & $-1,9 \pm 1,9$ & $-6,0$ & 0,0 \\
\hline Pós-operatório & $-1,6 \pm 0,9$ & $-3,0$ & 0,0 \\
\hline
\end{tabular}


Um dos principais obstáculos da cirurgia em pacientes jovens é a significativa e rápida resposta inflamatória pósoperatória, com subsequente aumento da incidência de $\mathrm{OCP}^{(16-17)}$. A importância da LIO na ocorrência da OCP, já é bem conhecida. As lentes acrílicas apresentam menor incidência de OCP do que as de polimetilmetacrilato ou de silicone. O desenho da óptica das lentes de acrílico dobráveis (Acrysof) também contribui para menor incidência de $\mathrm{OCP}^{(16)}$. Já alguns autores acreditam que a OCP está mais relacionada ao manejo intraoperatório da mesma, do que ao tipo de LIO utilizada ${ }^{(17)}$. Muitos estudos são necessários para investigar formas de reduzir a incidência de OCP na população pediátrica ${ }^{(17)}$. Na amostra estudada $71,4 \%$ apresentaram opacificação da cápsula posterior do cristalino, ocorrendo em média 1,2 $\pm 1,0$ anos após a cirurgia, tendo sido realizada capsulotomia posterior sem complicações adicionais.

Muitos estudos têm relatado efetiva expansão e estabilização do saco capsular, com o implante do anel endocapsular em casos de fragilidade zonular ${ }^{(10-15,18)}$.

Além disso, o anel endocapsular reduz a assimetria da tensão capsular, estabiliza a base vítrea, e previne a severa contração da cápsula posterior após a cirurgia ${ }^{(10)}$. Nos casos de severa fragilidade zonular, alguns autores sugerem suturar o anel endocapsular a esclera ${ }^{(2,11,13)}$. No entanto, em se tratando de uma fragilidade zonular generalizada e em alguns casos progressiva, pode haver deslocamento ou inclinação da LIO em função da tração exercida pela sutura ${ }^{(10,15)}$. A sutura do anel endocapsular em dois pontos opostos é uma opção nestes $\operatorname{casos}^{(10)}$. O presente estudo sugere uma técnica que mantém a integridade e previne a tensão no saco capsular, através do implante de uma lente rígida com uma das alças amputada. A alça íntegra contribui para a centralização da óptica, sem tensionar a zônula do lado oposto, onde se encontra a alça amputada apoiada sobre o anel endocapsular.

Os resultados obtidos no presente estudo sugerem que o implante primário de LIO com uma alça amputada é um procedimento viável, efetivo e com melhora da acuidade visual em todos os casos descritos. Não houve evolução da subluxação no decorrer do seguimento destes pacientes, apesar de se tratar de uma fragilidade progressiva da zônula, pelo contrário, observou-se uma estabilidade progressiva do conjunto saco capsular, anel endocapsular e LIO.

Em estudo comparando duas técnicas cirúrgicas diferentes para o tratamento da subluxação do cristalino, foi observado que a melhora significante da acuidade visual pós-operatória foi determinada principalmente pelos graus de subluxação pré-operatórios ${ }^{(18)}$. Estes achados alertam para os cuidados na seleção pré-operatória dos pacientes, fundamental para o sucesso da cirurgia.

Apesar de um dos casos descritos, com seguimento pósoperatório de apenas seis meses, ter apresentado melhora de três linhas (tabela de Snellen) na acuidade visual corrigida, e melhora do equivalente esférico de -11,50 DE no pré-operatório para-1,00 DE no pós-operatório, as indicações específicas, a segurança a longo prazo e eficácia, devem ser melhor avalia- das através de estudos, com amostras maiores e maior tempo de seguimento.

\section{CONCLUSÃO}

A técnica de implantação de LIO com uma alça amputada e apoiada sobre o anel endocapsular é uma opção no tratamento cirúrgico da subluxação congênita do cristalino com melhora da AV no pós-operatório, e estabilidade do conjunto saco capsular, anel endocapsular e LIO.

\section{ABSTRACT}

Purpose: To evaluate the postoperative results of congenital lens subluxation corrected by a new technique. Methods: Retrospective chart review of 21 eyes of 13 patients with no traumatic lens subluxation who underwent surgery in Altino Ventura Foundation from April, 1999 to April, 2004. The mean age was $8.7 \pm 5.4$ years old, and the mean follow-up period was $21.5 \pm 19.3$ months. Patients underwent phacoaspiration, endocapsular ring and intraocular lens (IOL) implantation. The implanted IOL had one loop haptic excised and was supported above the ring, inside the capsular bag promoting intraocular lens centralization. Results: Visual acuity improvement was observed in all cases. There was a significant reduction of the spherical equivalent and spherical component comparing the pre and postoperative refraction $(p<0.01)$. There was no statistically significant difference between the pre and postoperative cylinder component $(p=0.71)$. Posterior capsule opacification was a postoperative complication found in $71.4 \%$ of the cases. Early posterior capsulotomy was performed with no complications in these cases. Conclusion: Intraocular lens implantation with one loop haptic amputed and supported above the endocapsular ring is an option for the congenital lens subluxation surgical treatment, promoting lens centralization and postoperative visual acuity improvement.

Keywords: Lens subluxation; Prosthesis implantation; Marfan syndrome; Homocystinuria; Lenses, intraocular; Ectopia lentis/ surgery; Ophthalmologic surgical procedures; Visual acuity

\section{REFERÊNCIAS}

1. Anteby I, Isaac M, BenEzra D. Hereditary subluxated lenses visual performances and long-term follow-up after surgery. Ophthalmology. 2003;110(7):1344-8.

2. Severo NS, Kleinert F, Kwitko S. Conduta cirúrgica na subluxação do cristalino. Arq Bras Oftalmol. 2004;67(1):9-12.

3. Oliveira DF, Marchi PH, Arieta CEL. Resultados visuais no tratamento cirúrgico da subluxação de cristalino em crianças. Medicina (Ribeirão Preto). 2002; 35(1):62-9.

4. Neiva AG, Cunha RNP, Ferreira RC, Erwenne CM. Causas de cristalino ectópico em um hospital universitário. Arq Bras Oftalmol. 1995;58(5):307-9.

5. Tartarella MB, Araújo Filho A, Sallum JMF, Erwenne CM. Ectopia lentis et pupillae. Arq Bras Oftalmol. 1994;57(1):30-3.

6. Moreno-Montañés J, Sainz C, Maldonado MJ. Intraoperative and postoperative 
complications of Cionni endocapsular ring implantation. J Cataract Refract Surg. 2003;29(3):492-97.

7. Halpert M, BenEzra D. Surgery of the hereditary subluxated lens in children. Ophthalmology. 1996;103(4):681-6.

8. Vadalà $\mathrm{P}$, Capozzi P, Fortunato $\mathrm{M}$, DeVirgiliis E, VadalaF. Intraocular lens implantation in Marfan's syndrome. J Pediatr Ophthalmol Strabismus. 2000; 37(4):206-8. Comment in: J Pediatr Ophthalmol Strabismus. 2001;38(5):261.

9. Cionni RJ, Osher RH, Marques DM, Marques FF, Snyder ME, Shapiro S. Modified capsular tension ring for patients with congenital loss of zonular support. J Cataract Refract Surg. 2003;29(9):1668-73.

10. Cionni RJ, Osher RH. Management of profound zonular dialysis or weakness with a new endocapsular ring designed for scleral fixation. J Cataract Refract Surg. 1998;24(10):1299-306. Comment in: J Cataract Refract Surg. 2001;27(11): 1710-1.

11. Bhattacharjee H, Bhattacharjee K, Das D, Jain PK, Chakraborty D, Deka S. Management of a posteriorly dislocated endocapsular tension ring and a foldable acrylic intraocular lens. J Cataract Refract Surg. 2004;30(1):243-6.

12. Cionni RJ, Osher RH. Endocapsular ring approach to the subluxed cataractous lens. J Cataract Refract Surg. 1995;21(3):245-9. Comment in: J Cataract Refract Sureg. 2001;27(11):1710-1.
13. Lam DS, Young AL, Leung AT, Rao SK, Fan DS, Ng JS. Scleral fixation of a capsular tension ring for severe ectopia lentis. J Cataract Refract Surg. 2000; 26(4):609-12.

14. Dietlein TS, Jacobi PC, Konen W, Krieglstein GK. Complications of endocapsular tension ring implantation in a child with Marfan's syndrome. J Cataract Refract Surg. 2000;26(6):937-40.

15. Moreno-Montañés J, Sánchez-Tocino H, Rodriguez-Conde R. Complete anterior capsule contraction after phacoemulsification with acrylic intraocular lens and endocapsular ring implantation. J Cataract Refract Surg. 2002;28(4):717-9.

16. Müllner-Eidenböck A, Amon M, Moser E, Kruger A, Abela C, Schlemmer Y, et al. Morphological and functional results of Acrysof intraocular lens implantation in children. Prospective randomized study of age-related surgical management. J Cataract Refract Surg. 2003;29(2):285-93.

17. Ram J, Brar GS, Kaushik S, Gupta A, Gupta A. Role of posterior capsulotomy with vitrectomy and intraocular lens design and material in reducing posterior capsule opacification after pediatric cataract surgery. J Cataract Refract Surg. 2003; 29(8):1579-84

18. Waiswol M, Abujamra S, Cohen R, Almeida GV. Variação da acuidade visual em pacientes jovens com ectopia lentis submetidos à cirurgia. Arq Bras Oftalmol. 2005;68(4):495-504. 